\title{
Long-term response to ipilimumab after nivolumab failure in a case of anorectal melanoma with an intermediate tumor mutation burden and negative for PD-L1 expression
}

\author{
HITOMI SAKAI $^{1}$, MASAYUKI TAKEDA ${ }^{1}$, KAZUKO SAKAI $^{2}$, \\ KAZUTO NISHIO $^{2}$ and KAZUHIKO NAKAGAWA ${ }^{1}$ \\ Departments of ${ }^{1}$ Medical Oncology and ${ }^{2}$ Genome Biology, Kindai University Faculty of Medicine, \\ Osaka-Sayama, Osaka 589-8511, Japan
}

Received July 4, 2019; Accepted April 7, 2020

DOI: $10.3892 / \mathrm{mco} .2020 .2048$

\begin{abstract}
Anorectal melanoma is a rare disease with a poor prognosis and its response to immunotherapy remains poorly studied. The current study reports a case of recurrent anorectal melanoma in a 60-year-old woman that has exhibited a durable response to ipilimumab for $>2$ years. Given that the combination of nivolumab and ipilimumab was not approved for use in unresectable or metastatic melanoma at the time of presentation, the patient was initially treated with nivolumab monotherapy and switched to ipilimumab after nivolumab failure. The tumor was microsatellite stable, had an intermediate tumor mutation burden and was negative for programmed cell death-ligand-1 expression. However, the neutrophil-to-lymphocyte ratio in peripheral blood remained at $<5$ throughout the disease course. Although mucosal melanoma is not caused by ultraviolet radiation and has a lower mutation burden than cutaneous melanoma, the present case responded well to immunotherapy. Further evaluation of potential biomarkers for such patients is required.
\end{abstract}

\section{Introduction}

Mucosal melanoma is subtype of melanoma which arise from melanocytes in mucosal membranes. The major primary sites of mucosal melanoma are head and neck, anorectal, and female genital tract (1). Mucosal melanoma is typically diagnosed at a more advanced stage compared to cutaneous melanoma and generally carry a worse prognosis. Genome sequencing of mucosal melanomas revealed that they are not

Correspondence to: Dr Masayuki Takeda, Department of Medical Oncology, Kindai University Faculty of Medicine, 377-2 Ohno-higashi, Osaka-Sayama, Osaka 589-8511, Japan

E-mail: takeda_m@med.kindai.ac.jp

Key words: anorectal melanoma, mucosal melanoma, ipilimumab, nivolumab, tumor mutation burden, neutrophil-to-lymphocyte ratio markedly enriched in ultraviolet signature mutations, different from cutaneous melanoma (2).

Anorectal melanoma is a rare disease, accounting for only $1 \%$ of all anorectal malignancies, $1 \%$ of all melanomas and $18 \%$ of mucosal melanomas (3-5). The 5-year survival rate of anorectal melanoma was reported to be $<20 \%$ in the era before immunotherapy $(4,6)$, with this poor prognosis being related to early disease dissemination and a delay in diagnosis $(7,8)$. Clinically, it is sometimes misdiagnosed as hemorrhoids due to rectal bleeding. Furthermore, about $30 \%$ of anorectal melanomas are amelanotic and pathological diagnosis is difficult in those cases (9).

The only potentially curative option for mucosal melanoma is complete surgical resection with negative margins. Radiotherapy after surgery may be a treatment option, but there it has not been evaluated in prospective study (10). Given the limited treatment options available for individuals with metastatic mucosal melanoma, new therapies are urgently needed to improve prognosis. Immune checkpoint inhibitors (ICIs)-including the combination of nivolumab and ipilimumab-have recently become the standard treatment option for unresectable or metastatic melanoma regardless of tumor subtype, although treatment data for mucosal melanoma are limited compared with those for cutaneous melanoma (11-13). Here we report a case of advanced anorectal melanoma that progressed during nivolumab monotherapy but subsequently showed a durable response to ipilimumab.

\section{Case presentation}

A 60-year-old woman with a history of hemorrhoids for $>30$ years visited her local clinic with a complaint of hemorrhoid enlargement that had persisted for $>1$ year. Blood analysis revealed anemia, with a hemoglobin level of $6.5 \mathrm{~g} / \mathrm{dl}$. She underwent a hemorrhoidectomy, and the resected tissue was submitted for pathological examination because it had an atypical appearance. The pathological findings were suggestive of primary malignant melanoma. She was referred to our hospital 2 months after the surgery. Her Eastern Cooperative Oncology Group performance status was 0 . Digital examination and anoscopy did not detect any residual tumor. Chest 
and abdominal computed tomography $(\mathrm{CT})$ as well as positron emission tomography-CT also did not reveal residual tumor tissue or distant metastasis. Blood analysis, including the level of lactate dehydrogenase, showed no abnormalities. Histopathologic reevaluation revealed that atypical cells with enlarged nuclei were gathered in the stroma with a solid or alveolar pattern (Fig. 1) as well as the presence of brown pigment granules in the tumor. Immunostaining showed the tumor to be partly positive for vimentin, S-100, HBM-45, Melan A, and D2-40 as well as negative for cytokeratin, AE1/AE3, CD56, and chromogranin A. These findings were thus consistent with melanoma. No lymphatic invasion was evident by staining for the D2-40 lymphatic marker, and no vascular invasion was detected by CD31 immunostaining. The tumor proportion score (TPS) for programmed cell death-ligand-1 (PD-L1) was $<1 \%$ with PD-L1 antibody clone $28-8$. The tumor was also negative for the V600E mutation of $B R A F$. Next-generation sequencing analysis with the FoundationOne CDx panel, which detects mutations in 324 genes, select gene rearrangements, and genomic signatures including microsatellite instability and tumor mutational burden, revealed the tumor to be microsatellite stable (MSS) and to have a tumor mutation burden (TMB) of 8 mutations/Mb, a deletion of exons 28 to 37 of $N F 1$, an S37Y mutation of $C T N N B 1$, and an $\mathrm{R} 625 \mathrm{H}$ mutation of $S F 3 B 1$. These findings supported a diagnosis of $B R A F$ mutation-negative anorectal melanoma. Given the operation report and that hematoxylin-eosin staining of the excised tissue confirmed a surgical margin of $10 \mathrm{~mm}$, no additional surgery was performed. Six months after her first visit to our hospital, CT revealed that the patient had developed multiple lung and liver metastases (Fig. 2). Nivolumab monotherapy (3 mg every 2 weeks) was initiated. After six treatment cycles, chest and abdominal CT showed progression of liver metastasis (Fig. 2). Ipilimumab monotherapy ( $3 \mathrm{mg} / \mathrm{kg}$ every 3 weeks) was then started. After four cycles, the maximum number allowed, chest and abdominal CT confirmed a partial response. The treatment was well tolerated, with no immune-related adverse events. The response has been persisted over 32 months at the time of this writing (november 2019) (Fig. 2). The neutrophil-to-lymphocyte ratio (NLR) in peripheral blood has remained $<5$ throughout the disease course (Fig. 3).

\section{Discussion}

We here report a case of anorectal melanoma that has shown a long-lasting response to ipilimumab after failure of nivolumab treatment. The tumor was found to be MSS, to have an intermediate TMB, and to be negative for PD-L1 expression. Given that the combination of nivolumab and ipilimumab had not been approved for melanoma in Japan at the time the patient presented at our hospital, we initiated nivolumab monotherapy followed by ipilimumab monotherapy. A pooled analysis of patients with cutaneous $(n=665)$ or mucosal $(n=86)$ melanoma revealed a longer progression-free survival (PFS) and higher objective response rate for nivolumab in combination with ipilimumab than for nivolumab monotherapy (11). Among patients who received the combination therapy, the median PFS was 5.9 months for mucosal melanoma and 11.7 months for cutaneous melanoma, with objective response rates of 37.1 and $60.4 \%$, respectively. One reason for the poorer response of mucosal melanoma may be a lower TMB. The TMB, the total number of somatic mutations in a defined region of a tumor genome, is currently the most reliable predictive marker for ICI treatment in melanoma $(14,15)$. Two studies have shown that TMB as determined by whole-exome sequencing is related to the clinical benefit rate for ipilimumab monotherapy in melanoma $(16,17)$. A study based on next-generation sequencing with FoundationOne CDx for patients with advanced melanoma revealed the TMB to be high ( $>23.1$ mutations/Mb), intermediate (3.3-23.1 mutations/Mb), or low ( $<3.3$ mutations/Mb) in $27(41.5 \%), 24$ (36.9\%), and 14 (21.5\%) patients, respectively, with the TMB correlating with benefit from therapy targeted to the programmed cell death-1 (PD-1)-PD-L1 checkpoint (18). Furthermore, TMB in mucosal melanoma was found to be markedly lower than that in cutaneous melanoma, likely because of the contribution of ultraviolet-induced mutagenesis to cutaneous melanoma $(2,19,20)$.

PD-L1 expression has also been investigated as a potential biomarker for ICI therapy in melanoma. PD-L1 expression on tumor cells did not tend to be related to the response rate in melanoma patients treated with the combination of nivolumab and ipilimumab (21). As far as we are aware, the relation between PD-L1 expression and response to ipilimumab monotherapy has not been examined. With regard to PD-L1 positivity in mucosal melanoma, a small study found that, with a TPS of $\geq 5 \%$ as the cutoff, the proportion of tumors positive for PD-L1 was 44\% (16/36), a value similar to that for cutaneous melanoma at $35 \%$ (19/54) $(22,23)$.

The present case was found to be MSS, with an intermediate TMB, and negative for PD-L1 expression. These characteristics of an 'immunologically cold tumor' would be expected to confer a low sensitivity to ICIs. A recent report showed that many mucosal melanoma cases had PD-L1 low/negative disease, and average mutational load of 4 cases was much lower than that of cutaneous melanoma (24). Consistent with these findings, our tumor immune profile showed low PD-L1 expression as well as low TMB; however, there is a discrepancy the efficacy of ICI. The reason for the discrepancy between the biological features of the tumor and the clinical benefit conferred by ipilimumab in the current case is unclear. However, a high NLR in peripheral blood has been shown to be strongly associated with a poor outcome of ipilimumab treatment in patients with advanced melanoma (25-28). An NLR of $\geq 4$ before initiation of ipilimumab treatment was thus associated with a worse overall survival compared with a ratio of $<4$ in patients with metastatic melanoma (25). An NLR of $\geq 5$ at each time point examined was also associated with a worse overall survival, PFS, and response to ipilimumab treatment (26). In the present case, the NLR was $<5$ at baseline and remained so during and after ipilimumab treatment. The potential of the NLR as a biomarker for ipilimumab treatment in melanoma patients thus warrants further investigation.

In conclusion, the present case shows that ipilimumab is a potentially effective treatment option for patients with metastatic mucosal melanoma including anorectal melanoma, even though mucosal melanoma in general has been found to have a less favorable outcome during ipilimumab treatment compared with cutaneous melanoma. Even if tumor histology, past treatment history, and certain biomarkers suggest that a tumor is immunologically cold, it might still respond to ICI 

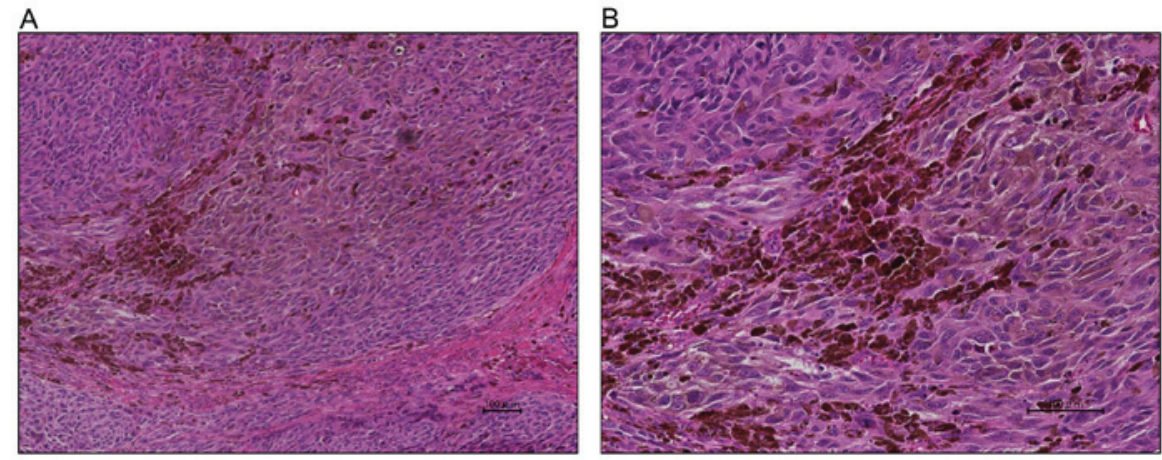

Figure 1. Histopathologic analysis of the surgical specimen. (A) Hematoxylin-eosin staining of the tumor revealed a solid or alveolar pattern. (B) Higher magnification revealed that the tumor was composed of atypical cells with enlarged nuclei and high mitotic activity as well as the presence of brown pigment granules. Scale bars, $100 \mu \mathrm{m}$.
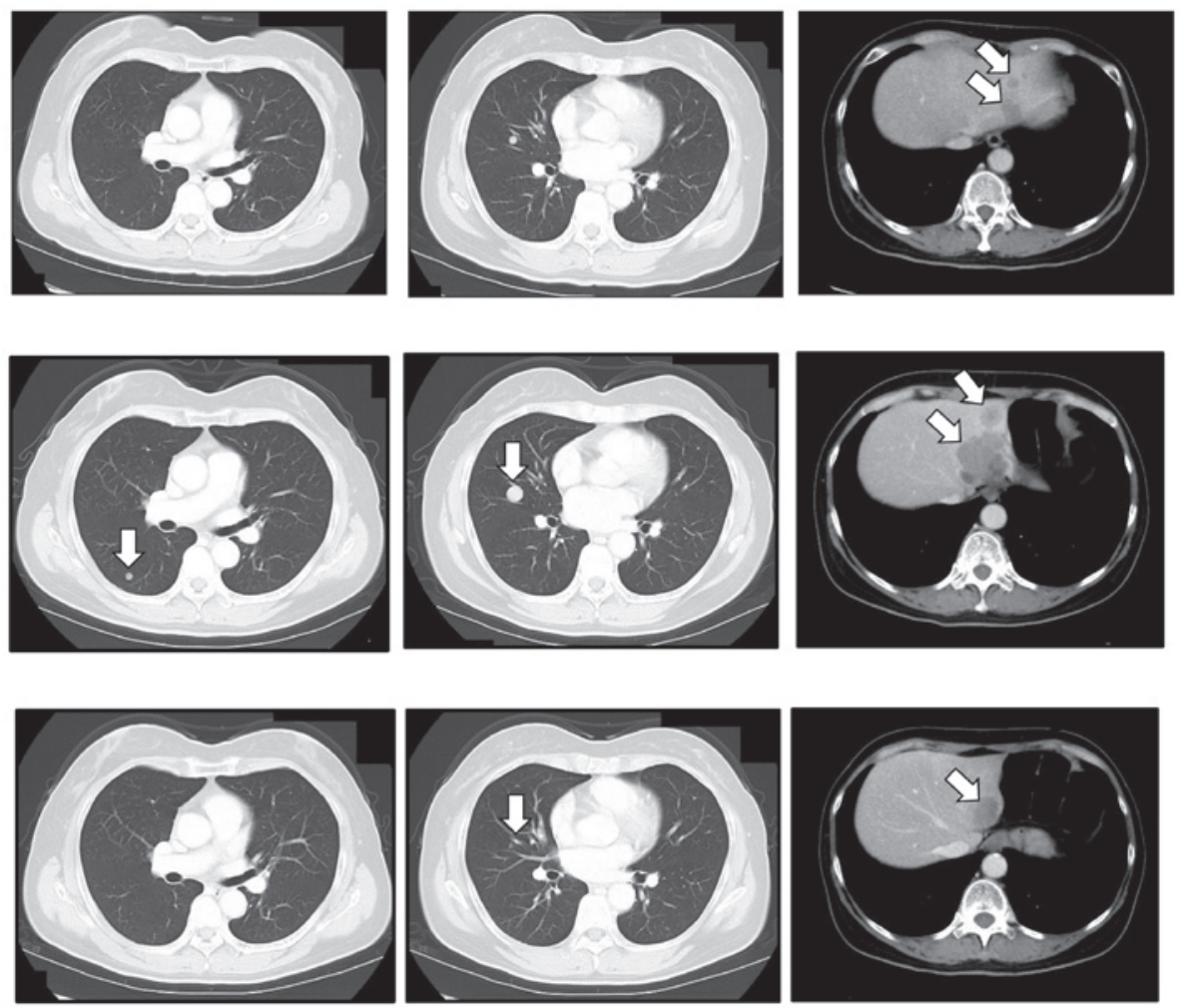

Figure 2. Computed tomography images of the patient obtained before initiation of nivolumab treatment (upper), before initiation of ipilimumab treatment (middle) and 2 years after the initiation of ipilimumab treatment (lower). Arrows indicate metastases from anorectal melanoma.

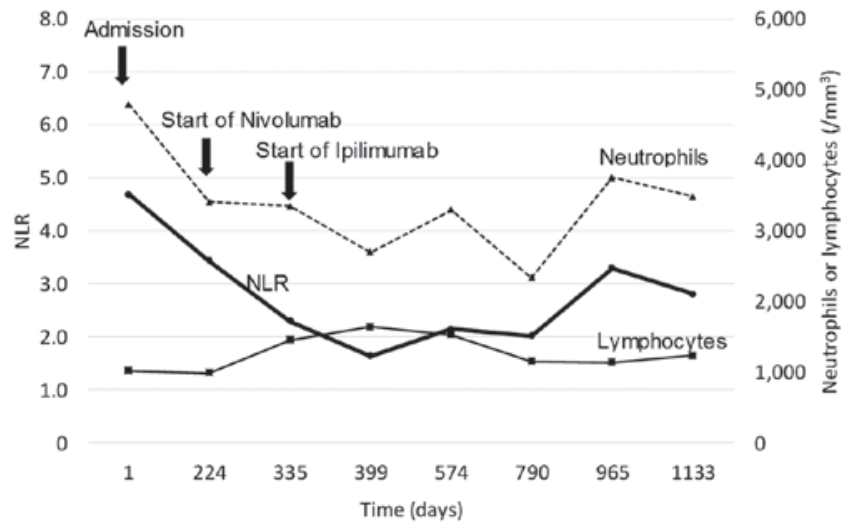

Figure 3. Time course of neutrophil and lymphocyte counts in peripheral blood as well as the NLR for the patient. NLR, neutrophil-to-lymphocyte ratio. treatment. Evaluation of the NLR should be considered before excluding ICI therapy as an option.

\section{Acknowledgements}

Not applicable.

\section{Funding}

No funding was received.

\section{Availability of data and materials}

All data generated or analyzed during the present study are included in this published article. 


\section{Authors' contributions}

HS, KS, KNa, KNi and MT conceived the current study. HS drafted the current study HS, MT, KS, KNa and KNi acquired and analyzed the data. HS, MT, KS, KNi and KNa wrote, reviewed and edited the manuscript. All authors read and approved the manuscript and agree to be accountable for all aspects of the research in ensuring that the accuracy or integrity of any part of the work are appropriately investigated and resolved.

\section{Ethics approval and consent to participate}

All procedures performed in studies involving human participants were in accordance with the ethical standards of the institutional and/or national research committee and with the 1964 Helsinki declaration and its later amendments or comparable ethical standards. Informed consent was obtained from the patient.

\section{Patient consent for publication}

Written informed consent was obtained from the patient for publication of the present case report and accompanying images prior to receiving chemotherapy.

\section{Competing interests}

The authors declare that they have no competing interests.

\section{References}

1. Lian B, Cui CL, Zhou L, Song X, Zhang XS, Wu D, Si L, Chi $\mathrm{ZH}$, Sheng XN, Mao LL, et al: The natural history and patterns of metastases from mucosal melanoma: An analysis of 706 prospectively-followed patients. Ann Oncol 28: 868-873, 2017.

2. Furney SJ, Turajlic S, Stamp G, Nohadani M, Carlisle A, Thomas JM, Hayes A, Strauss D, Gore M, van den Oord J, et al: Genome sequencing of mucosal melanomas reveals that they are driven by distinct mechanisms from cutaneous melanoma. J Pathol 230: 261-269, 2013.

3. van Schaik PM, Ernst MF, Meijer HA and Bosscha K: Melanoma of the rectum: A rare entity. World J Gastroenterol 14: 1633-1635, 2008.

4. Chang AE, Karnell LH and Menck HR: The national cancer data base report on cutaneous and noncutaneous melanoma: A summary of 84,836 cases from the past decade. The American college of surgeons commission on cancer and the American cancer society. Cancer 83: 1664-1678, 1998.

5. Haanen JBAG: Converting cold into hot tumors by combining immunotherapies. Cell 170: 1055-1056, 2017.

6. Brady MS, Kavolius JP and Quan SH: Anorectal melanoma. A 64-year experience at memorial sloan-kettering cancer center. Dis Colon Rectum 38: 146-151, 1995.

7. Perez DR, Trakarnsanga A, Shia J, Nash GM, Temple LK, Paty PB, Guillem JG, Garcia-Aguilar J, Bello D, Ariyan C, et al: Locoregional lymphadenectomy in the surgical management of anorectal melanoma. Ann Surg Oncol 20: 2339-2344, 2013.

8. Hicks CW, Pappou EP, Magruder JT, Gazer B, Fang S, Wick EC, Gearhart SL, Ahuja N and Efron JE: Clinicopathologic presentation and natural history of anorectal melanoma: A case series of 18 patients. JAMA Surg 149: 608-611, 2014.

9. Hillenbrand A, Barth TF, Henne-Bruns D and Formentini A: Anorectal amelanotic melanoma. Colorectal Dis 10: 612-615, 2008.

10. Malaguarnera G, Madeddu R, Catania VE, Bertino G, Morelli L, Perrotta RE, Drago F, Malaguarnera M and Latteri S: Anorectal mucosal melanoma. Oncotarget 9: 8785-8800, 2018.
11. D'Angelo SP, Larkin J, Sosman JA, Lebbé C, Brady B, Neyns B, Schmidt H, Hassel JC, Hodi FS, Lorigan P, et al: Efficacy and safety of nivolumab alone or in combination with ipilimumab in patients with mucosal melanoma: A pooled analysis. J Clin Oncol 35: 226-235, 2017.

12. Del Vecchio M, Di Guardo L, Ascierto PA, Grimaldi AM,Sileni VC, Pigozzo J, Ferraresi V, Nuzzo C, Rinaldi G, Testori A, et al: Efficacy and safety of ipilimumab $3 \mathrm{mg} / \mathrm{kg}$ in patients with pretreated, metastatic, mucosal melanoma. Eur J Cancer 50: 121-127, 2014.

13. Postow MA, Luke JJ, Bluth MJ, Ramaiya N, Panageas KS, Lawrence DP, Ibrahim N, Flaherty KT, Sullivan RJ, Ott PA, et al: Ipilimumab for patients with advanced mucosal melanoma. Oncologist 18: 726-732, 2013.

14. Büttner R, Longshore JW, López-Ríos F, Merkelbach-Bruse S, Normanno N, Rouleau E and Penault-Llorca F: Implementing TMB measurement in clinical practice: Considerations on assay requirements. ESMO Open 4: e000442, 2019.

15. Hugo W,Zaretsky JM, Sun L, Song C, Moreno BH,Hu-Lieskovan S, Berent-Maoz B, Pang J, Chmielowski B, Cherry G, et al: Genomic and transcriptomic features of response to Anti-PD-1 therapy in metastatic melanoma. Cell 165: 35-44, 2016.

16. Van Allen EM, Miao D, Schilling B, Shukla SA, Blank C, Zimmer L, Sucker A, Hillen U, Foppen MHG, Goldinger SM, et al: Genomic correlates of response to CTLA-4 blockade in metastatic melanoma. Science 350: 207-211, 2015.

17. Snyder A, Makarov V, Merghoub T, Yuan J, Zaretsky JM, Desrichard A, Walsh LA, Postow MA, Wong P, Ho TS, et al: Genetic basis for clinical response to CTLA-4 blockade in melanoma. N Engl J Med 371: 2189-2199, 2014.

18. Johnson DB, Frampton GM, Rioth MJ, Yusko E, Xu Y, Guo X, Ennis RC, Fabrizio D, Chalmers ZR, Greenbowe J, et al: Targeted next generation sequencing identifies markers of response to PD-1 blockade. Cancer Immunol Res 4: 959-967, 2016.

19. Berger MF, Hodis E, Heffernan TP, Deribe YL, Lawrence MS, Protopopov A, Ivanova E, Watson IR, Nickerson E, Ghosh P, et al: Melanoma genome sequencing reveals frequent PREX2 mutations. Nature 485: 502-506, 2012.

20. Hayward NK, Wilmott JS, Waddell N, Johansson PA, Field MA, Nones K, Patch AM, Kakavand H, Alexandrov LB, Burke $\mathrm{H}$, et al: Whole-genome landscapes of major melanoma subtypes. Nature 545: 175-180, 2017.

21. Larkin J, Chiarion-Sileni V, Gonzalez R, Grob JJ, Cowey CL, Lao CD, Schadendorf D, Dummer R, Smylie M, Rutkowski P, et al: Combined nivolumab and ipilimumab or monotherapy in untreated melanoma. N Engl J Med 373: 23-34, 2015.

22. Kaunitz GJ, Cottrell TR, Lilo M, Muthappan V, Esandrio J, Berry S, Xu H, Ogurtsova A, Anders RA, Fischer AH, et al: Melanoma subtypes demonstrate distinct PD-L1 expression profiles. Lab Invest 97: 1063-1071, 2017.

23. Taube JM, Anders RA, Young GD, Xu H, Sharma R, McMiller TL, Chen S, Klein AP, Pardoll DM, Topalian SL and Chen L: Colocalization of inflammatory response with B7-h1 expression in human melanocytic lesions supports an adaptive resistance mechanism of immune escape. Sci Transl Med 4: 127ra37, 2012.

24. Dodds TJ, Wilmott JS, Jackett LA, Lo SN, Long GV, Thompson JF and Scolyer RA: Primary anorectal melanoma: Clinical, immunohistology and DNA analysis of 43 cases. Pathology 51: 39-45, 2019.

25. Zaragoza J, Caille A, Beneton N, Bens G, Christiann F, Maillard $\mathrm{H}$ and Machet L: High neutrophil to lymphocyte ratio measured before starting ipilimumab treatment is associated with reduced overall survival in patients with melanoma. Br J Dermatol 174: 146-151, 2016.

26. Cassidy MR, Wolchok RE, Zheng J, Panageas KS, Wolchok JD, Coit D, Postow MA and Ariyan C: Neutrophil to lymphocyte ratio is associated with outcome during ipilimumab treatment. EBioMedicine 18: 56-61, 2017.

27. Ferrucci PF, Gandini S, Battaglia A, Alfieri S, Di Giacomo AM, Giannarelli D, Cappellini GC, De Galitiis F, Marchetti P, Amato G, et al: Baseline neutrophil-to-lymphocyte ratio is associated with outcome of ipilimumab-treated metastatic melanoma patients. Br J Cancer 112: 1904-1910, 2015.

28. Ferrucci PF, Ascierto PA, Pigozzo J, Del Vecchio M, Maio M, Antonini Cappellini GC, Guidoboni M, Queirolo P, Savoia $\mathrm{P}$, Mandalà $\mathrm{M}$, et al: Baseline neutrophils and derived neutrophil-to-lymphocyte ratio: Prognostic relevance in metastatic melanoma patients receiving ipilimumab. Ann Oncol 27: 732-738, 2016. 